\title{
Comparative Study of Ultraviolet and Infrared Femtosecond Laser Irra- diation on Textile Polymers PET and PA66
}

\author{
Erieta-Katerina Koussi ${ }^{1}$, Hyeon Jin Jung ${ }^{1,2}$, Nicolas Faure ${ }^{1}$, Christophe Donnet ${ }^{1}$, Cyril Mauclair ${ }^{1,3}$, and Xxx Sedao*1,3 \\ ${ }^{1}$ Université de Lyon, Université Jean Monnet - Saint-Etienne, CNRS, Institute of Optics Graduate \\ School, Laboratoire Hubert Curien, UMR 5516 CNRS, 42023 Saint-Etienne, France \\ ${ }^{2}$ Electronic Convergence Division, Korea Institute of Ceramic Engineering \& Technology (KICET), \\ Jinju 52851, South Korea \\ ${ }^{3}$ GIE Manutech-USD, Saint-Etienne 42000, France \\ *Corresponding author'se-mail: xxx.sedao@univ-st-etienne.fr
}

\begin{abstract}
Femtosecond lasers emit short pulses whose temporal width is in the range of less than a picosecond to a few femtoseconds (fs), thereby enabling extremely high peak-power machining with minimum thermal damages. Herein, we employed femtosecond laser pulses as a versatile tool for surface processing of 2 polymers commonly used in textile industry, Polyethylene terephthalate (PET), and Polyamide66 (PA66). This work focuses on a comparison of ultraviolet (UV, $257 \mathrm{~nm}$ ) and infrared (IR, $800 \mathrm{~nm}$ ) femtosecond laser irradiation at the surface of the polymers PET and PA66. The polymers are modified topographically and chemically, by direct laser irradiation, through different ablation routines, with direct bond breaking for UV fs laser irradiation, and multiphoton absorption for IR fs laser irradiations. The interaction strongly depends on the laser beam operation conditions, such as laser fluence, number of pulses, wavelength etc, as illustrated by post mortem analysis based on optic microscopy, Scanning Electron Microscopy and Raman spectroscopy. This work defines the change in the characteristics of the polymers as a function of the laser wavelengths. UV fs laser processing appears to be more advantageous, offering a large process window and a high precision in ablation.
\end{abstract}

DOI: $10.2961 /$ jlmn.2020.03.2015

Keywords: femtosecond laser, laser wavelength, laser fluence, polyethylene terephthalate (PET), polyamide66 (PA66, nylon66)

\section{Introduction}

A variety of experimental techniques, such as lithography, electro-position, mechanical polishing, continuous laser or lasers with long pulses, allows the preparation of surfaces with controlled roughness and hence desired functionalities [1-3]. However, most of these techniques have intrinsic limitations in terms of speed, precision, repeatability, unwanted side effects (wear, thermal and chemical degradation).

Herein, ultrashort femtosecond laser ablation is an important process in micro- and nano- machining of industry $[4,5]$. After a decade of technology development, femtosecond laser has emerged as a convenient technique for the manufacturing of materials. Ultrafast laser pulses can interact with materials at extremely localized dimensions and often negligible amount of undesired thermal effect. As a consequence, ultrashort laser pulses have already been used in high precision micro- and nano- machining, especially in metals [6] and semiconductors [7]. However, in terms of polymers, due to their dielectric structure and inhomogeneity, the machining precision is somewhat less impressive. Accordingly, they have attracted the interest of numerous researchers in the past years and a lot progress has been made. Due to these characteristics, ultrafast laser pro- cessing has become industrially viable technology in some fields such as microelectronics and cellar phones, and is considered a close future industry solution in other fields, namely textiles and leatherwares [8]. This potential, in turn, demands more attention and research efforts $[9,10]$.

In this work, we report a study on the surface modification of two polymers, Polyethylene terephthalate (PET), and Polyamide66 (PA66), by means of ultrafast laser ablation. At present, synthetic fibers are being developed rapidly, and have become the main stream material in textile industry [11]. PET, and PA66 are among the most common widespread thermoplastic polymer resins of the polyester and polyamide groups. It is used for clothing, bedding, other textile and containers for liquids and foods, thermoforming for manufacturing, and other industrial fiber products. The performance and stability properties of the laser irradiated polymer are key issues towards laser surface modifications and potentially functionalization of these two polymers, for traceability, aesthetic appearance, liquid repellent and anti-bacteria etc [12]. However, very few studies have been reported on this particular subject of matter $[13,14]$. Consequently, herein we communicate our results on ultrafast laser irradiation of PET and PA66. The ultimate goal is to explore the potential of surface function- 
alization of these polymers [2, 15-17, 20-24]. Therefore, we performed our experiments with femtosecond laser irradiation in IR $(800 \mathrm{~nm})$ regime as a standard approach, as well as an "out-of-the-ordinary" irradiation condition with femtosecond laser in UV $(257 \mathrm{~nm})$. The objective was to evaluate the spatial resolution achievable with different laser wavelengths, as well as possible process window within which material degradation due to laser irradiation would not be a major issue.

\section{Experimental details}

\subsection{Materials}

In this study, the PET and PA 66 sheets were produced by injection molding, and then the surface underwent a polishing process step, with a final roughness $\mathrm{Ra}=600 \mathrm{~nm}$. The rectangular sample's dimensions were $25 \times 15 \times 2 \mathrm{~mm}$ for the experiments. Fig. 1 displays the polymers' chemical structures. The PET chain consists of an aromatic ring and carbonyl groups. The PET is a general-purpose thermoplastic polymer which belongs to the polyester family of polymers. Semi-crystalline polyester commonly used in packaging and fiber applications. Polyester resins are known for their excellent combination of properties such as mechanical, thermal, chemical resistance as well as dimensional stability. The glass transition temperature, melting point and boiling point of $\mathrm{PET}$ are $72^{\circ} \mathrm{C}, 250^{\circ} \mathrm{C}$ higher and $350^{\circ} \mathrm{C}$ respectively and if the temperature rises further than that, the polymer gets decomposed [16]. Likewise, the PA66 chain consists of an amide groups and an ethyl group. The PA66, commercially known as Nylon, is one of the most popular engineering thermoplastics. PA66 is synthesized by polycondensation of hexamethylenediamine and adipic acid (two monomers each containing 6 carbon atoms). PA66 has a glass transition temperature around $70{ }^{\circ} \mathrm{C}$ and its melting point is $264^{\circ} \mathrm{C}$ [16].

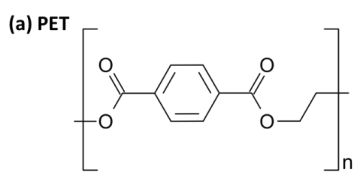

(b) PA66

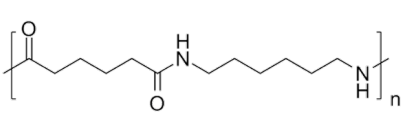

Fig. 1 The chemical structure of (a) Polyethylene terephthalate (PET), and (b) Polyamide 66 (PA66).

\subsection{Femtosecond laser surface modification}

By $\mathrm{D}^{2}$ experiment we determined the ablation thresholds for the two PET and PA66 polymers, in ultraviolet (UV $257 \mathrm{~nm}$ ) and infrared (IR 800nm) radiation. The spot size was measured with an optical microscope [17, 25].

For laser pulses with a Gaussian spatial beam profile, the diameter $\mathrm{D}$ of the ablation zone and the maximum laser energy $\mathrm{E}_{0}$ are

$$
\mathrm{D}^{2}=2 \omega_{0}^{2} \ln \left(\mathrm{E}_{0} / \mathrm{E}_{\mathrm{th}}\right)
$$

$\omega_{0}$ is $1 / \mathrm{e}^{2}$ beam radius. Using a graph of square diameters as a function of the pulse energy logarithm, the Gaussian $\omega_{0}$ beam radius was estimated due to the linear relationship between pulsed energy $\mathrm{E}$ and the laser fluence $\mathrm{F}_{0}$.

The maximum laser fluence $F_{0}$ in the Gaussian beam radius and the pulse energy $\mathrm{E}$ can be calculated as

$$
\mathrm{F}_{\text {th }}=2 \mathrm{E}_{\text {th }} / \pi \omega_{0}^{2}
$$

By plotting $\mathrm{D}^{2}$ as a function of $\ln \left(\mathrm{E}_{0}\right)$, the laser spot size can be obtained from the gradient and the critical fluence from the intersection with the energy axis. The Gaussian spot radius of $\omega_{0}=9.02 \mu \mathrm{m}$ in UV determined by the $\mathrm{D}^{2}$ method. Likewise, The Gaussian spot radius $\omega_{0}=18.83$ $\mu \mathrm{m}$ in was derived by the same method.

\subsubsection{UV laser irradiation conditions}

The impact area on the polymers were produced using a femtosecond laser system (Pharos from Light Conversion) equipped with a $4^{\text {th }}$ harmonics generation module (model Hiro from the same company). The laser has a central wavelength of $1030 \mathrm{~nm}$ with a pulse duration of $190 \mathrm{fs}$ and a maximum pulse energy at $2 \mathrm{~mJ}$. After the $4^{\text {th }}$ harmonic generator, the wavelength of the emitting light is centered at $257 \mathrm{~nm}$ in the UV band. Before delivery onto the polymers sample surface, the laser pulses were linearly polarized and then attenuated through a pair of neutral density filters. The laser beam is normally focused, through an achromatic lens on the sample that is vertically mounted on an $\mathrm{X}-\mathrm{Y}$ motorized translation stage. The dimension of the beam spot on the surface of the sample, $\omega_{0}=9.02 \mu \mathrm{m}\left(1 / \mathrm{e}^{2}\right.$ intensity), is extrapolated with the single shot (on polished silicon wafers) D-square method [17]. The morphological structures on the polymer surfaces were determined by the laser fluence and the number of pulses, which were varied from $0.086 \mathrm{~J} / \mathrm{cm}^{2}$ to $2.936 \mathrm{~J} / \mathrm{cm}^{2}$ and from 1 to 5 pulses. Detailed fs laser experiment conditions are presented in Table 1 below.

\subsubsection{IR laser irradiation conditions}

The impact area on the polymers substrate was produced using a Ti: Sapphire femtosecond laser system (Legend Coherent Inc.). The laser has a central wavelength of $800 \mathrm{~nm}$ with a pulse duration of $50 \mathrm{fs}$ and a repetition rate of $2 \mathrm{kHz}$ with a maximum pulse energy at $3 \mathrm{~mJ}$. The laser irradiation peak fluence was varied between $1.796 \mathrm{~J} / \mathrm{cm}^{2}$ and $16.167 \mathrm{~J} / \mathrm{cm}^{2}$, and the number of pulses from 1 to 500 . The dimension of the beam spot radius on the surface of the sample, $\omega_{0}=18.83 \mu \mathrm{m}$, was determined by the same method that mentioned above. The setup was similar to the ultraviolet laser experiments, and detailed setup descriptions can be found in our earlier work [18].

\subsection{Surface characterization}

The morphological analysis of the laser irradiated polymers was performed using an optical microscope (Axioscope 5, ZEISS Routine Microscopy), and a scanning electron microscope (FEI, NovaNanoSEM 200). In order to investigate the surface chemistry, Raman analysis was carried out before and after laser irradiation. The Micro-

Table 1 Detailed laser conditions at UV and IR

\begin{tabular}{ccc}
\hline$\lambda$ & $\lambda=257 \mathrm{~nm}$ & $\lambda=800 \mathrm{~nm}$ \\
\hline$\omega_{0}$ & $9.02 \mu \mathrm{m}$ & $18.83 \mu \mathrm{m}$ \\
$\mathrm{F}$ & $0.09-3 \mathrm{~J} / \mathrm{cm}^{2}$ & $1.8-16 \mathrm{~J} / \mathrm{cm}^{2}$ \\
$\mathrm{~N}$ pulses & $1-5$ & $10-500$ \\
$\tau$ & $190 \mathrm{fs}$ & $50 \mathrm{fs}$ \\
\hline
\end{tabular}


Raman spectrometer (LabRAM ARAMIS, HR800, HORIBA Jobin-Yvon) with the Raman laser beam options at $325 \mathrm{~nm}, 422 \mathrm{~nm}, 488 \mathrm{~nm}$, and $633 \mathrm{~nm}$ wavelengths, obtained the spectra with a spectral resolution of 9 or $1 \mathrm{~cm}^{-1}$ with a beam splitter as an excitation source at room temperature. They were recorded for all samples in the $1000-$ $3500 \mathrm{~cm}^{-1}$ regions.

\section{Results}

The sections of this article are organized in the following order: firstly, the evaluation of the ablation thresholds, then the morphology inspection and finally the analysis of surface chemistry. In each section, two subsections are attributed to UV and IR, respectively, in a comparative manner.

\subsection{Ablation thresholds}

\section{1. 1. UV fs laser irradiation}

Given the high energy of photons $(\sim 6 \mathrm{eV})$ of fs laser pulses of $257 \mathrm{~nm}$, comparable to the bonding energies of the polymers, the onset of surface modification was observed even in single shot experiments, under certain high pulse energy conditions. Fig. 2 presents the D-square extrapolation of laser spots size as well as the ablation thresholds for the two polymers. These graphs are made based on 5-shot experiments. The ablation threshold for PA66 is $F_{\text {th }}$ $=0.2 \mathrm{~J} / \mathrm{cm}^{2}$. For PET the ablation threshold is 3 times smaller, $F_{\text {th }}=0.06 \mathrm{~J} / \mathrm{cm}^{2}$. The difference may be due to the presence of the chromophores in PA66, or possibly higher molecular weight of PA66 over PET leading to the formation of highly viscous molten material during ablation [26].

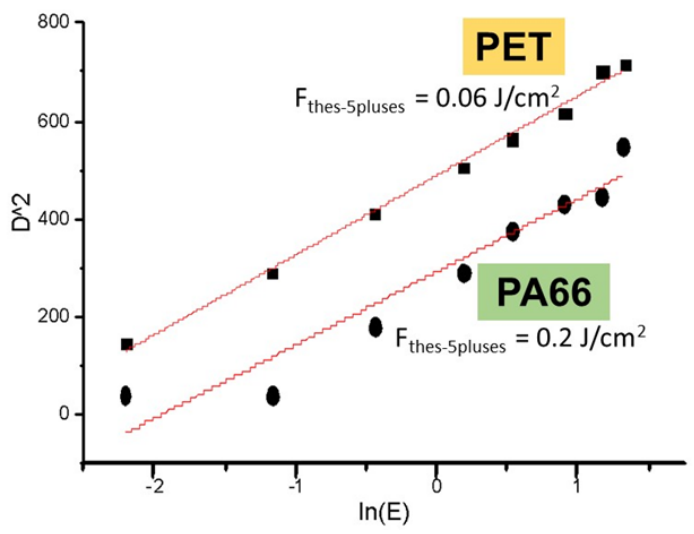

Fig. 2 D-square $\left(D^{2}\right)$ extrapolation of UV ablation thresholds for PA66 and PET, 5 laser pulses.

\section{1. 2. IR fs laser irradiation}

Compared to the UV counterpart, the IR photon energy of $\sim 1 \mathrm{eV}$ is much smaller than the bandgap of the polymer bonds. The ablation is considered to be through multiphoton absorption. Furthermore, for a very large range of laser pulse energies, a big number of laser pulses was necessary to achieve visible surface modifications on PET and PA66 (especially in the later). In order to get a reasonable number of measurable craters for the data extrapolation, 500 pulses were applied for both polymers. Fig. 3 presents the Dsquare extrapolation of laser spot sizes as well as the ablation thresholds for the two polymers. These graphs are made based on 500-shot experiments. According to the graph, not only the pulse number is greater than the UV case, but the ablation thresholds are also much higher. The threshold for PA66 is $F_{\text {th }}=7.9 \mathrm{~J} / \mathrm{cm}^{2}$. For PET the ablation threshold is about 5 times smaller, $F_{\text {th }}=1.5 \mathrm{~J} / \mathrm{cm}^{2}$. It is also worthwhile to note here, since this discussion is involved in the later discussion, that the ablation threshold of PET at 25 pulses was determined to be $F_{t h}=4.5 \mathrm{~J} / \mathrm{cm}^{2}$. For the sake of simplicity and cleanliness, the data extrapolation for this process condition is not plotted in Fig. 3.

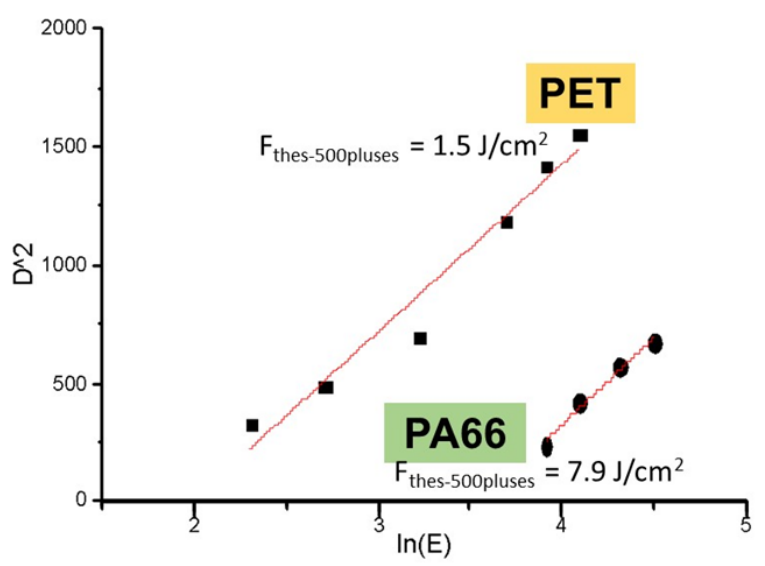

Fig. 3 D-square $\left(D^{2}\right)$ extrapolation of IR ablation thresholds for PA66 and PET, 500 laser pulses.

\section{2. Morphology evolution}

\section{2. 1. UV fs laser irradiation}

The FE-SEM images of the PET and PA66 polymer surfaces irradiated with single-shot pulses at different laser fluences are shown in Fig. 4 and Fig. 5. These are the images observed under various laser fluences. The laser fluences are (a) $2.936 \mathrm{~J} / \mathrm{m}^{2}$, (b) $2.505 \mathrm{~J} / \mathrm{m}^{2}$, (c) $1.918 \mathrm{~J} / \mathrm{m}^{2}$, and (d) $1.331 \mathrm{~J} / \mathrm{m}^{2}$.

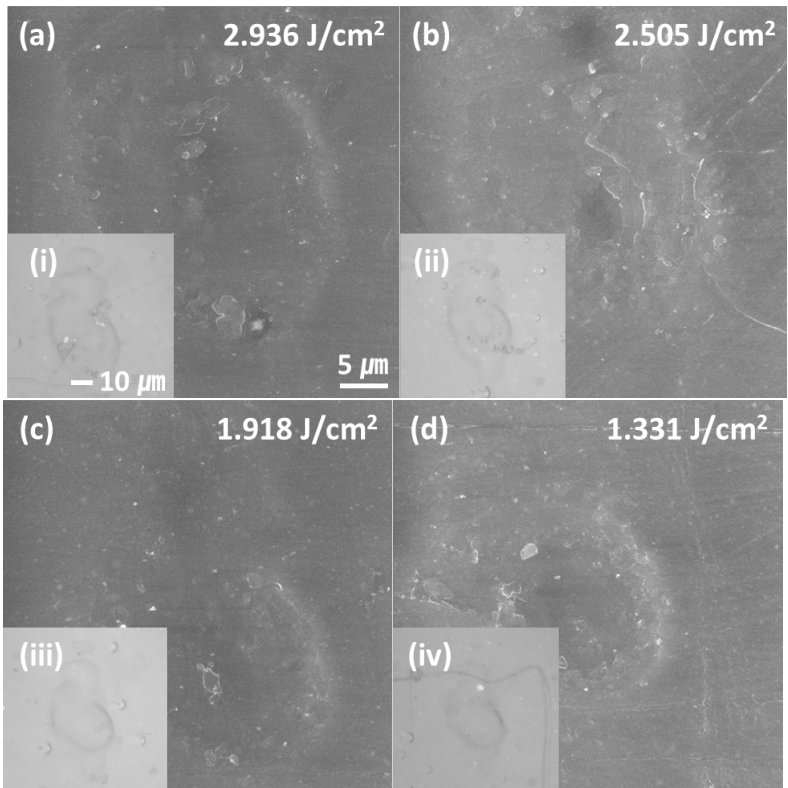

Fig. 4 PET irradiated by 5 UV fs pulses: (a) - (d) FE-SEM images, and at the lower corner of each SEM micrograph, the insets optical microscope images (i) - (iv) corresponding to laser fluence variance from $2.936-1.331 \mathrm{~J} / \mathrm{cm}^{2}$, respectively 
The number of pulses was 5 , as explained in the previous section. In the case of PET, the craters appear to be smooth, featureless, even without sharp edges at the peripheric. On the contrary, the craters observed on PA66 have a greater contrast, with some random features inside the craters. The edges of the craters are clearly defined. Some small particles are located around these craters, which are thought to be re-depositions.

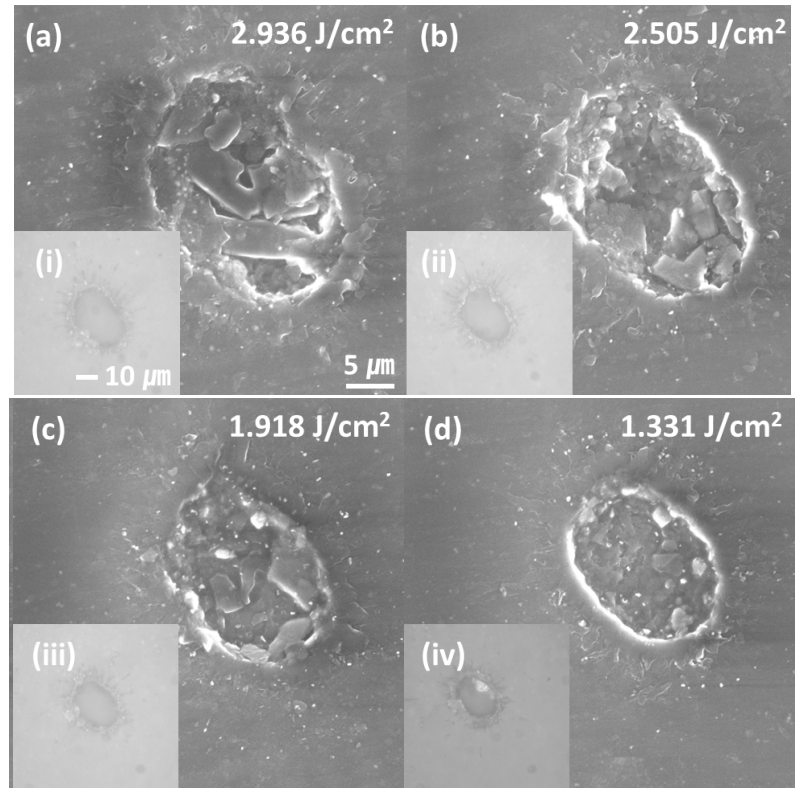

Fig. 5. PA66 irradiated by 5 UV fs pulses: (a) - (d) FE-SEM images, and in the lower corner of each SEM micrograph, the insets of optical microscope images (i) - (iv) corresponding to the laser

fluence variation from $2.936-1.331 \mathrm{~J} / \mathrm{cm}^{2}$, respectively.

\section{2. 2. IR fs laser irradiation}

After the IR femtosecond laser interaction, surface observation was required to characterize the morphologies of each polymer surface. The morphology development was quite similar for PET and PA66. Therefore, for the sake of simplicity and being representative, only typical SEM micrographs of PET are presented here, in Fig. 6. These impacts were made with 25 laser pulses. For a laser fluence smaller than $5.389 \mathrm{~J} / \mathrm{cm}^{2}$, no visible marks on PET were identified under SEM inspection. The observation with SEM at higher magnification was difficult, due to the wellknown fact of charge accumulation effect on the insulator polymer surfaces. It has been shown elsewhere that such observation remains complicated even with environmental SEM [19]. Nonetheless, the SEM images shown in Figure 6 reveal readily a great deal about the interaction between PET and fs laser pulses in IR. At the lower laser fluence of $5.389 \mathrm{~J} / \mathrm{cm}^{2}$, the crater is without a clearly defined form, suggesting a non-linear nature of the ablation onset. The size of the craters increases by increasing the laser fluence. As observed, the higher the laser fluence, the more the craters shape follows the Gaussian profile of the incident laser. The craters are perceived to be deep - supposedly related to the greater penetration depth of the IR laser light into the PET.
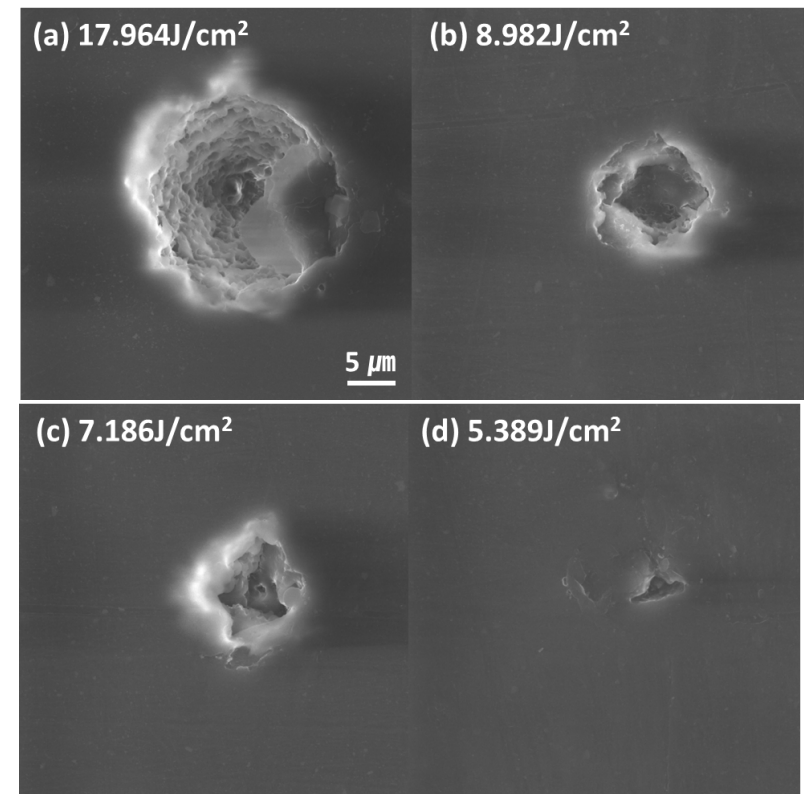

Fig. 6. PET irradiated by 25 IR fs pulses: (a) - (d) FE-SEM images corresponding to laser fluence variation from $17.964-5.389$ $\mathrm{J} / \mathrm{cm}^{2}$, respectively.

\section{3. Raman studies}

The Raman analysis is a powerful mean for identification of molecules and intra-molecular bonds as well as polymers. In order to identify the most sensitive measurement regime, trials of the spectrum acquisition were made to virgin PET and PA66 substrates at different sensing wavelengths, from $325 \mathrm{~nm}$ to $633 \mathrm{~nm}$. The wavelengths at 633 $\mathrm{nm}$ and $422 \mathrm{~nm}$ appeared to excite a significant number of characteristic peaks for the two polymers, therefore, they were selected as the excitation sources for the analysis presented in the following sections.

\section{3. 1. UV fs laser irradiation}

The PET and PA samples irradiated at $2.936 \mathrm{~J} / \mathrm{cm}^{2}$ with 5 pulses were evaluated. For a fair comparison, for instance, to avoid mis-interpretation due to inhomogeneity issues between different polymer substrates, each time a Raman spectrum was acquired from non-irradiated polymer surface at the vicinity of the laser impacts. In Fig. 7 (a) the Raman spectra of the non-irradiated PA66 is depicted. Fig. 7 (b), depicts the spectrum of the PA66 obtained from the laser irradiation. The Raman frequency shifts of various peaks, in an ascending order, are 1137, 1239, 1306, 1381, $1390,1448,1489,1644,2878,2928,3311 \mathrm{~cm}^{-1}$. The peak at $3311 \mathrm{~cm}^{-1}$ is due to the $\mathrm{N}-\mathrm{H}$ stretching of the amide $\mathrm{A}$. The peak at $1644 \mathrm{~cm}^{-1}$ is due to the $\mathrm{C}=\mathrm{O}$ stretching of amide I group [20-22], while the peak at $1306 \mathrm{~cm}^{-1}$ is assigned to $\mathrm{CH}_{2}$ twisting mode. The peaks at 1448,2878 and at 2928 $\mathrm{cm}^{-1}$ are assigned to $\mathrm{CH}_{2}$ bending, symmetric and asymmetric stretching modes, respectively. However, Raman spectra observed all these peaks in the PA66 polymer decreased significantly after laser irradiation, which is consistent with some significant chemical bond alteration induced by laser irradiation. It is suggested that the physical or chemical properties of the PA66 changed due to the reaction of the laser irradiated surface. Such change, which implies possible degradation in the performance of the ma- 
terials (color, strength, ductility, elasticity etc), is considered to be undesirable.

Raman spectra were also obtained for PET polymer without laser processing, as shown in Fig. 7 (c). The Raman spectra of PET forms a strong fluorescence background covering certain peaks. The Raman frequency shifts of various peaks in an increasing order are 1120, 1188, $1298,1620,1733,2971,3090 \mathrm{~cm}^{-1}$. The intensity of the carbonyl peak at $1733 \mathrm{~cm}^{-1}$ was large, indicating that the compound was an ester. The peak at $16201 \mathrm{~cm}^{-1}$ is due to the ring mode $8 \mathrm{a}$ of the aromatic ring. The weak peak of $3090 \mathrm{~cm}^{-1}$ was produced by the vibration of the benzene ring skeleton ${ }^{23} 24$. Raman spectra of UV fs laser irradiated PET (Fig. 7(d)) exhibit no changes compared to the reference. It can be expected that there is little change in physical or chemical properties under such laser irradiation condition (intense laser fluence).

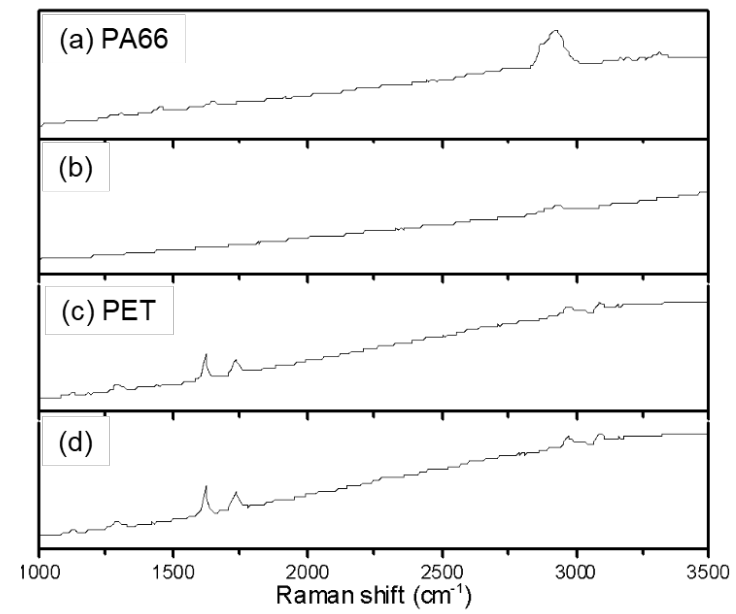

Fig. 7. The Raman spectra of the PET, PA66 surface before (a), (c) and after (b), (d) the UV fs pulse laser irradiation $(\lambda=257 \mathrm{~nm})$. The average laser fluence and the number of pulses were 2.936 $\mathrm{J} / \mathrm{cm}^{2}$ and 5 pulses. Raman probing source was $422 \mathrm{~nm}$.

\section{3. 2. IR fs laser irradiation}

The PET surface with laser impacts achieved with a series of different laser fluences was analyzed. As mentioned in the morphological study section above, high number of pulses make deep holes and such process condition is not well suited for the intended application, neither practical for the surface characterization. For these reasons, the PET impacts with 25 pulses were taken to the analysis. In Fig. 8 the Raman spectra, obtained at $633 \mathrm{~nm}$ probing wavelength, of the non-irradiated PET was depicted. Spectra (b) - (d), show the spectrum of the PET obtained at different powers from the IR fs laser irradiation. The Raman frequency shifts of various peaks in a sequential order are 1121, 1179, $1289,1618,1727,2966$, and $3085 \mathrm{~cm}^{-1}$. The intensities of all peaks are relatively low and weak. It is found nonetheless the carbonyl peak at $1727 \mathrm{~cm}^{-1}$ and the peak associated with the benzene ring at $1618 \mathrm{~cm}^{-1}$ are distinguishable and characteristic. These peaks are observed to disappear as the laser fluence increases. The onset of this degradation is readily observed at a relatively low fluence level of 5.389 $\mathrm{J} / \mathrm{cm}^{2}$, which is just slightly above about the 25-pulse threshold of $4.5 \mathrm{~J} / \mathrm{cm}^{2}$. Due to this effect (and by surface morphology observation), we suggest that PET is more suitable to be UV fs laser processed rather than IR one.

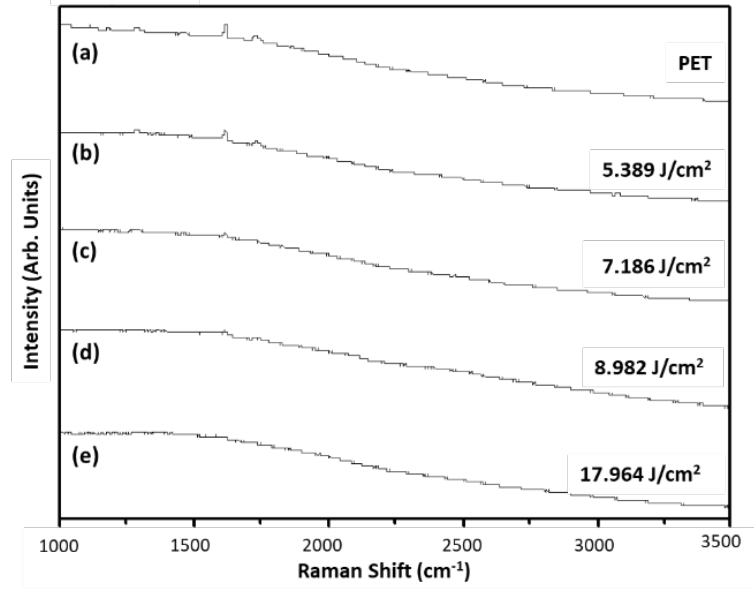

Fig. 8. The Raman spectra of the PET surface before (a) and after pulsed laser irradiation $(\mathrm{b})$ - $(\mathrm{e})$ with the IR fs laser irradiation $(\lambda$ $=800 \mathrm{~nm}, \tau=50 \mathrm{fs}, \mathrm{N}=25$ ). The Raman spectra of the PET depend on the laser fluence (b) $5.389 \mathrm{~J} / \mathrm{cm}^{2}$, (c) $7.186 \mathrm{~J} / \mathrm{cm}^{2}$ (d) $8.982 \mathrm{~J} / \mathrm{cm}^{2}$, and (e) $17.964 \mathrm{~J} / \mathrm{cm}^{2}$, respectively. Raman probing source was $633 \mathrm{~nm}$.

Given the experience gained from the above-mentioned PET IR fs laser irradiation analysis, for PA66, in order not to drill deep holes, nor prompt onset of surface chemistry degradation, we decided to optimize our experiment strategy by fixing at a fair moderate fluence, i.e. a fluence close to the ablation threshold, and checking the spectrum evolution with increasing number of laser pulses. Raman spectrum was obtained, at a Raman probing wavelength of 633 $\mathrm{nm}$, for PA66 polymer, as shown in Fig. 9(a), before the laser irradiation, and (b) - (d) after the laser irradiation. For greater numbers of laser pulses ( $>50$, for instance), the craters were too big and deep to allow a reasonable Raman analysis to be carried out on them. As a consequence, it was impossible to obtain a dissent Raman spectrum from the laser irradiated impacts under these conditions. The peaks from virgin PA66 are similar to those obtain previously in section 3.3.1. The peaks approximately from 1000 to $1200 \mathrm{~cm}^{-1}$ are assigned to $\mathrm{C}-\mathrm{C}$ stretching mode such as skeletal vibration $[20,22]$. We observed that this peak group disappears as the number of laser pulses increased. In particular, laser irradiation at the 50 pulses reduces the intensity of the peak as a whole, making the C-C stretching mode almost invisible. Given that the laser fluence is just slight above the 500-pulse laser ablation threshold for PA66, it has to be admitted that the process window for PA66 at IR is really small. 


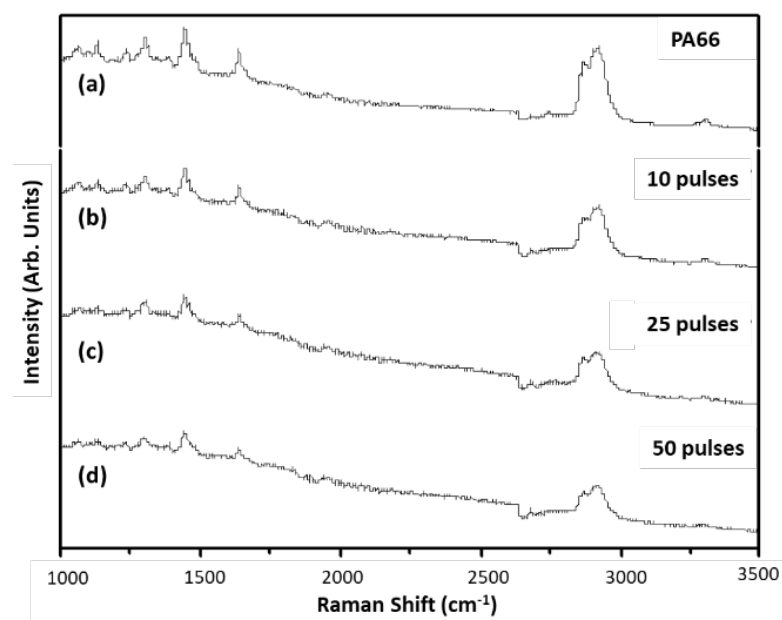

Fig. 9. The Raman spectra of the PA66 surface before (a) and after (b) - (d) IR laser irradiation at the laser fluence $8.982 \mathrm{~J} / \mathrm{cm}^{2}$. The number of pulses applied to the sample was 10,25, and 50 respectively. Raman probing source was at $633 \mathrm{~nm}$.

\section{Discussions}

As one could expect, we observed that the ablation thresholds of the polymers in question are very susceptible to the wavelength of the laser source. This is very likely due to the difference in photon energy at different laser wavelengths, and the correlation between the photon energy and band gap energy of the polymers. In UV condition, the surface modifications are shallow, ideal for surface modification for added surface functionalities. Furthermore, the onset of the surface modification could be attained at a small number of laser pulses, this is favorable in terms of its transferability from laboratory process to industrial scale. Nonetheless, surface chemistry analysis indicates precautions may have to be taken, when operated at high laser fluences, for example, two magnitude greater than the threshold (see PA66 in Figs. 2 and 7), to avoid substantial chemical degradation of the material. To that end, PET is more resistant to UV irradiation - an intense fluence to introduce noticeable degradation is far higher than its ablation threshold. All these observations assure in reality a pretty safe process window for the polymers processing with UV fs laser pulses. In order to be able to treat large surface areas of the polymers, an UV Galvano system enabling raster scanning of the UV laser pulses is being built in house at this moment.

In IR fs laser processing, a relatively moderate laser fluence could readily induce surface degradation. As reported above, chemistry change identified even when the laser fluence is near the ablation threshold. To boot, due to the longer penetration depths, and/or multiphoton ionization nature of the ablation, the IR fs laser processing tends to produce high aspect ratio hole type features, which is far from the initial goal of surface functionalization through surface modification. Nonetheless, this weak linear absorption characteristic of IR fs laser is a unique advantage for bulk processing of such materials for different applications [27].

Obviously, the current study is merely a glimpse at the possible chemical reactions/degradations induced by laser irradiation. More detailed reaction pathways and products could be identified through in-depth studies related to the binding energy of polymers and the bonds responses to laser photons at difference wavelengths, in which more dedicated analysis means and/or approaches could be deployed, such as time-of-flight based analysis, pump-probe spectroscopy, and/or X-ray photoelectron spectroscopy based top surface layer analysis [28]. More insight from these surface analyses may lead to other surface functionalization alternatives, such as laser assisted grafting [29]. Nevertheless, this report raises attention to the potential of UV fs laser pulses, although more complicated to operate (more non-linear conversions involving higher harmonic generation, less optics to choose from, higher risk potential of accidence such as skin burns), could act as a more versatile and powerful tool in polymer surface engineering.

\section{Conclusions}

In summary, we observed optical and chemical changes on the surface of PET and PA66 polymers using UV and IR ultrashort laser pulse. They have different surface morphologies and chemical decomposition results depending on the wavelength of the laser and the type of polymers. Presumably, due to a high photon energy at $257 \mathrm{~nm}$, the UV fs laser pulses produce shallow and smooth craters on these polymers. On the other hand, presumably due to the transparency in the near infrared regime of the two polymers, IR fs laser pulses tend to drill deep holes. Our research also shows the process window, defined by ablation threshold and decomposition laser does, is large for UV fs laser processing compared to IR processing, for both PET and PA66. Based on these observations, we could suggest that, at least for the intended applications in clothing, textiles and packaging, the UV fs laser processing is more suitable for surface modification of these polymers. However, more dedicated studies are required in order to explain the optimal processing conditions with the associated mechanisms for UV or IR laser processing without damaging the material surroundings.

\section{Acknowledgments and Appendixes}

This work was supported by the FUI project "Deperflex II".

\section{References}

[1] S. Sarbada and Y.C. Shin: Appl. Surf. Sci., 405, (2017) 465.

[2] S. Moradi, S. Kamal, P. Englezos, and S.G. Hatzikiriakos: Nanotech., 41, (2013) 415302.

[3] A.F. Lasagni, M. Bieda, T. Roch, and D. Langheinrich; Laser Technik Journal, 8, (2011) 45.

[4] A.F. Lasagni, C. Gachot, K. Trinh, M. Hans, A. Rosenkranz, T. Roch, S. Eckhardt, T. Kunze, M. Bieda, D. Günther, V. Lang, and F. Mücklich: Proc. SPIE, Vol. 10097, (2017) 1009211.

[5] K. Sugioka and Y. Cheng: Light Sci. Appl., 3, (2014) 149.

[6] A. Vorobyev and C. Guo: Opt. Express, 14, (2006) 2164.

[7] S. Abreu Fernandes, B. Schoeps, K. Kowalick, R. Nett, C. Esen, M. Pickshaus, and A. Ostendorf: Physics Procedia, 41, (2013) 802.

[8] M. Malinauskas, A. Žukauskas, S. Hasegawa, Y. Hayasaki, V. Mizeikis, R. Buividas, ans S. Juodkazis: Light: Sci \& Appl., 5, (2016) 16133. 
[9] T. Kunze, C. Zwahr, B. Krupop, S. Alamri, F. Rößler, and A. F. Lasagni: Proc. SPIE, Vol. 10092, (2017) 212

[10] D. Guenther, J. Valle, S. Burgui, C. Gil, C. Solano, A. Toledo-Arana, R. Helbig, C. Werner, I. Lasa, and A.F. Lasagni: Proc. SPIE, Vol. 9736, (2016) 973611.

[11] S. Alamri and A. F. Lasagni: Opt. Express, 25, (2017) 9603.

[12] I. Martín-Fabiani, E. Rebollar, S. Pérez, D. R. Rueda, M. C. García-Gutiérrez, A. Szymczyk, Z. Roslaniec, M. Castillejo, and T. A. Ezquerra: Langmuir, 28, (2012) 7938

[13] C.W. Kan: J. Appl. Polym. Sci., 107, (2008) 1584.

[14] C.W. Kan and C.C. Wong: Fibers Polym., 14, (2013) 230.

[15] S. Alamri, A.I. Aguilar-Morales, and A. F. Lasagni: European Polymer Journal, 99, (2018) 27.

[16] C. DeArmitt and R.Rothon: "Applied Plastics Engineering Handbook". (Processing and Materials, 2011) 864.

[17] J. M. Liu: Opt. Lett., 7, (1982) 196.

[18] X. Sedao, C. Maurice, F. Garrelie, J-P. Colombier, S. Reynaud, R. Quey, G. Blanc, and F. Pigeon: Appl. Surf. Sci., 302, (2014) 114.

[19] S. Hammouti, B. Beaugiraud, M. Salvia, C. Mauclair, A. Pascale-Hamri, S. Benayoun, and S. Valette: Appli. Surf. Sci., 327, (2015) 277.

[20] J. V. Miller and E. G. Bartick: Appl Spect., 55, (2001) 1729.

[21] E.L. Papadopoulou, F. Pignatelli, S. Marras, L. Marini, A. Davis, A. Athanassioua, and I. S. Bayer: RSC Advances, 6, (2016) 6823.

[22] C. Menchaca, A. Alvarez-Castillo, G. MartinezBarrera, H. Lopez-Valdivia, H. Carrasco, and V. M. Castano: International Journal of Materials and Product Technology, 19, (2003) 521.

[23] C. Zhu, N. Tong, L. Song, and G. Zhang: Proc. SPIE, Vol. 9656, (2015), 96560E.

[24] E. Rebollar, S. Pérez, M. Hernández, C. Domingo, M. Martín, T.A. Ezquerra, J.P. García-Ruizc, and M. Castillejo: Phys. Chem. Chem. Phys., 16, (2014) 17551.

[25] B. S. Haq, H. U. Khan, K. Alam, M. Mateenullah, S. Attaullah, and I. Zari: Appl. Opt., 54, (2015) 7413.

[26] S. Ravi - Kumar, L. Benjamin, X. Zhang, L. Hao, H. Lyu, and Q. Hantang: Polym. Int., 68, (2019) 1391.

[27] M. Malinauskas, A. Žukauskas, S. Hasegawa, Y. Hayasaki, V. Mizeikis, R. Buividas, and S. Juodkazis: Light Sci. Appl., 5, (2016) e16133.

[28] M. Peng, L. Li, J. Xiong, K. Hua, S. Wang, and T. Shao: Coatings, 7, (2017) 123.

[29]M. Charbonnier, M. Alami, M. Romand, J.P. Girardeau-Montaut, and M. Afif: Appl. Surf. Sci., 109-110, (1997) 206-211.

(Received: June 26, 2020, Accepted: December 2, 2020) 